1 The Roles of Different Design Techniques in Learning Tactical Scenes of Play through

2 Dynamic Visualizations: A Brief Review

3 Ghazi Rekik ${ }^{1 *}$, Yosra Belkhir ${ }^{1,2}$, Mohamed Jarraya ${ }^{3}$, Mohamed Amine Bouzid ${ }^{1,3}$, Yung-

4 Sheng Chen ${ }^{4}$, and Cheng-Deng Kuo ${ }^{5,6,7}$ *

51 Research Laboratory: Education, Motricité, Sport et Santé, EM2S, LR19JS01, Sfax

6 University, Sfax, Tunisia; ghazi.rek@gmail.com (G.R.)

$7{ }^{2}$ High Institute of Sport and Physical Education, Manouba University, Tunis, Tunisia;

$8 \quad$ belkhir.ysr@gmail.com (Y.B.)

$9 \quad{ }^{3}$ High Institute of Sport and Physical Education, Sfax University, Sfax, Tunisia; jarrayam@yahoo.fr (M.J.); bouzid.mohamed-amine@hotmail.fr (M.A.B.)

${ }^{4}$ Department of Exercise and Health Sciences, University of Taipei, Taipei 111, Taiwan;

12 yschen@utaipei.edu.tw (Y.S.C.)

$13{ }^{5}$ Department of Medical Research, Taipei Veterans General Hospital, Taipei 112, Taiwan;

14 cdkuo23@gmail.com (C.D.K.)

$15{ }^{6}$ Department of Internal Medicine, Taian Hospital, Taipei 104, Taiwan

${ }^{7}$ Tanyu Research Laboratory, Taipei 112, Taiwan

*Correspondence: ghazi.rek@gmail.com; cdkuo23@gmail.com 


\section{The Roles of Different Design Techniques in Learning Tactical Scenes of Play through}

\section{Dynamic Visualizations: A Brief Review}

Abstract: Dynamic visualizations have been developed to exchange information that transforms over time across a broad range of professional and academic contexts. However, these visual tools may impose substantial demands on the learner's cognitive resources that are very limited in current knowledge. Cognitive load theory has been used to improve learning from dynamic visualizations by providing certain design techniques to manage learner cognitive load without adding any oral/written explanations. This systematic review examined a series of experimental studies assessing the roles of these design techniques in learning tactical scenes of play through dynamic visualizations. Electronic databases PubMed and Google Scholar were used to search relevant articles. Eleven studies were eventually included for the systematic review based on the eligibility criteria. The present review revealed that adapting design techniques to the level of learners' expertise, type of depicted knowledge, and level of content complexity is a crucial part of effective learning.

Keywords: Cognitive load theory, Dynamic visualizations, Design techniques, Learning, Team sports.

\section{(1)}

0

4

(1) 3 44 45 


\section{Introduction}

\subsection{Learning from dynamic visualizations}

Dynamic visualizations are external representations that change over time and represent a nonᄀ-stop flow of perceptual information, yielding an illusion of movements [1, 2]. These instructional visualizations could be as animations used for communicating descriptive information/knowledge [3, 4], or as video clips used for presenting motor knowledge/skills $[5,6]$. The use of dynamic visualizations in a learning environment can present numerous benefits. Firstly, they seem to be the most natural visual tool to convey dynamic properties (e.g., translation, transformation) that are tricky to describe verbally [7]. Secondly, they can depict dynamic information in an explicit and continuous way, which may help the observer to establish appropriate internal representation [8]. Thirdly, they can show the micro-steps of the dynamic phenomenon, while offering a concrete and global view [9], and avoiding the process of mental inference [10]. Fourthly, recent findings indicated that using dynamic visualizations in instructional contexts could be relevant for improving learners' attitudes such as motivation and engagement [11-13].

Despite the advantages of dynamic visualizations in learning, the Cognitive Load Theory (CLT: $[14,15])$ argued that dynamic visualizations may impose substantial demands for the learner's cognitive resources that are very limited in both capacity and duration, which might hinder learning [16]. The CLT is a theory that considers how visual information impacts on working memory (WM) and learning. According to this theory, learning from dynamic visualizations depends specifically on two categories of cognitive load. The first category is "the intrinsic cognitive load" which is dependent upon the levels of content complexity. From a cognitive load viewpoint, dealing with simple dynamic visualization (i.e., content with a little number of interactive elements) consumes less WM resources and leads to easier learning. In contrast, dealing with complex dynamic visualization (i.e., content with 
an excessive number of interactive elements) consumes large amounts of WM resources and makes learning difficult [17]. The second category is "the extraneous cognitive load" which is related to the designed instructional materials that interfere with schema acquisition. In this framework, it is suggested that the transient nature of information is responsible for the increase of extraneous cognitive load when learning from dynamic visualizations (the transient information effect) $[15,18]$. Indeed, videos or animations provide a transient, nonpermanent stream of information that vanishes from the computer screen [14]. Consequently, learners are obliged to process current information while simultaneously trying to maintain the previously given information and integrate it with novel information in long term memory [3, 19]. Overall, to improve learning from dynamic visualizations, a number of design techniques have been proposed to manage learner cognitive loads (intrinsic and extraneous cognitive loads) without adding any oral/written explanations.

\subsection{Dynamic visualizations and design techniques}

On one hand, research within cognitive load theory suggested two design techniques which effectively enable the control/management of intrinsic cognitive load [14].

The first technique is to employ sequential presentation [e.g., 20]. This instructional strategy recommends presenting information depicted in dynamic visualization serially rather than concurrently. This method may be relevant for learning as it provides learners with less information to be concurrently treated in working memory and thus, facilitates the integration of information in long term memory [21, 22]. In addition, the sequential presentation of the dynamic visualizations' components in a defined order could refer to a form of temporal cueing, facilitating the building of ordered knowledge in long term memory [20].

The second technique is the prediction method. This strategy pushes learners to anticipate/predict future macro/micro steps of dynamic visualizations. This mental process is supposed to improve learning from dynamic representations as it encourages learners to 
activate their acquired knowledge of the system and/or help them to realize what they do not know about the system and stimulate a greater focus [10].

On the other hand, researches in the scope of cognitive load theory suggested five design techniques (without adding any oral/written explanations) which effectively enable the reduction of extraneous cognitive load caused by the transient nature of dynamic visualizations $[14,15]$.

The first technique is the use of static visualizations [e.g., 2, 13, 23, 24]. This method consists of replacing videos or animations with a series of static pictures or with a static diagram, describing the essential states of the dynamic system. This instructional strategy may decrease the extraneous cognitive load investment by allowing learners to benefit from sufficient time to identify and process relevant information and effectively integrate it in long term memory $[25,26]$. Moreover, using static visualizations, compared to dynamic representations, offer the possibility to revise and compare different parts of the display as frequently as desired [27].

The second technique is to employ segmentation [e.g., 28, 29]. The segmentation of videos/animations corresponds to an insertion of pauses or time breaks between the key segments/steps of the dynamic phenomenon. This strategy provides learners with supplementary time to process and assimilate information received in the previous segments without having to simultaneously attend the next incoming information [29]. Moreover, this method could be referred to as temporal cueing, because it allows learners to distinguish between macro/micro dynamic events in the display [30].

The third technique is the incorporation of cues/signals [e.g., 31, 32]. This instructional strategy can be applied either by "adding elements" such as arrows, lines, and thick frames, or "without adding elements" via coloring, flashing and zooming [1]. According to the cognitive load theory, using cues or signals, especially without adding elements, in 
dynamic visualizations may improve learning because they are able to highlight the crucial information elements and thereby, to direct the learner's attention towards it [33, 34].

The fourth technique is the decrease of presentation speed [e.g., 17, 35]. This method consists of reducing the number of frames per second. Decreasing presentation speed of dynamic visualizations may provide learners with additional time to achieve the required cognitive processing in WM, while reducing the probability that key information is missing [36]. Moreover, such design technique is beneficial as it reduces the perceptual/cognitive demands by allowing learners to build a mental representation of local parts (i.e., micro/macro dynamic events), which then can be integrated into a coherent mental model [17, 37].

The fifth technique is the use of learner-control [e.g., 38, 39]. This instructional design allows learners to control the dynamic display through interactive features such as stopping, replaying, reversing or changing speed. Using this method in computer-based learning environments allows learners to repeat and process the missed part of the display. Furthermore, this user-control give an additional time for learners to process, consolidate and transfer information into long term memory before proceeding to the next segment/step [39].

\subsection{The present study}

A synthesis of the literature about how dynamic visualizations should be designed may be helpful for coaches and Physical education teachers in order to guarantee an effective learning of tactical scenes of play. However, a literature review about this topic has not been published until today. This paper reviews a series of experimental studies examining the roles of certain design techniques without adding any oral/written explanations in learning game systems through dynamic visualizations.

\section{Method}

This systematic review was conducted and reported in accordance with the guidelines of the preferred reporting items for systematic reviews and/or meta-analysis (PRISMA) [40]. 


\subsection{Search strategy}

Scholarly electronic databases (PubMed and Google Scholar) were searched without applying any time limits or filters; the final search being completed on August 27th, 2020. Moreover, we performed manual searches of relevant journals and reference lists obtained from published articles. Electronic databases were searched using a range of combinations between the following descriptors: "animation", "video", “dynamic visualization", “dynamic representation", “design techniques", “instructional design”, “team sports”, “tactical learning", “recall accuracy", “decision making”, “comprehension", “performance”. Two researchers (G.R. and Y.B.) independently considered each of the located articles for its appropriateness for inclusion. In case of uncertainty, discussion with a third researcher (M.J.) determined the final inclusion or exclusion of the article.

\subsection{Inclusion criteria}

To be suitable for inclusion, studies had to fulfill the following selection criteria: (a) studies focused solely on the role of design techniques on learning tactical scenes of play through any type of dynamic visualization (i.e., video or animation); (b) studies recruiting male and female subjects at any age category and competitive level in sports; (c) studies based on purely visual learning environment (i.e., without adding any oral/written explanations) in order to avoid the occurrence of modality effect (for this point see [41, 42]); (d) studies involving cognitive load and/or learning measurements; (e) original studies written in English and published in peer-reviewed journals.

\subsection{Exclusion criteria}

Studies not meeting with the following criteria were excluded: (a) studies based on multimedia learning environment (i.e., combination of visual and oral/written explanations); (b) proceedings, conference papers, thesis, reviews, book chapters, books, expert interviews, 
171 meta-analysis, or commentary articles; (c) articles not written in English; (d) articles not

172 published in peer-reviewed journals.

173 3. Results

174

\subsection{Study selection}

The search strategies yielded a preliminary pool of 253 possible papers. Subsequently, 39 duplicate articles were removed. The full text of 48 articles were retrieved and assessed for eligibility based on the inclusion criteria. After a careful review of their full texts, 37 articles were excluded and the remaining 11 articles (published between 2013 and 2020 in peerreviewed journals) were eligible for inclusion in the review (Figure 1).

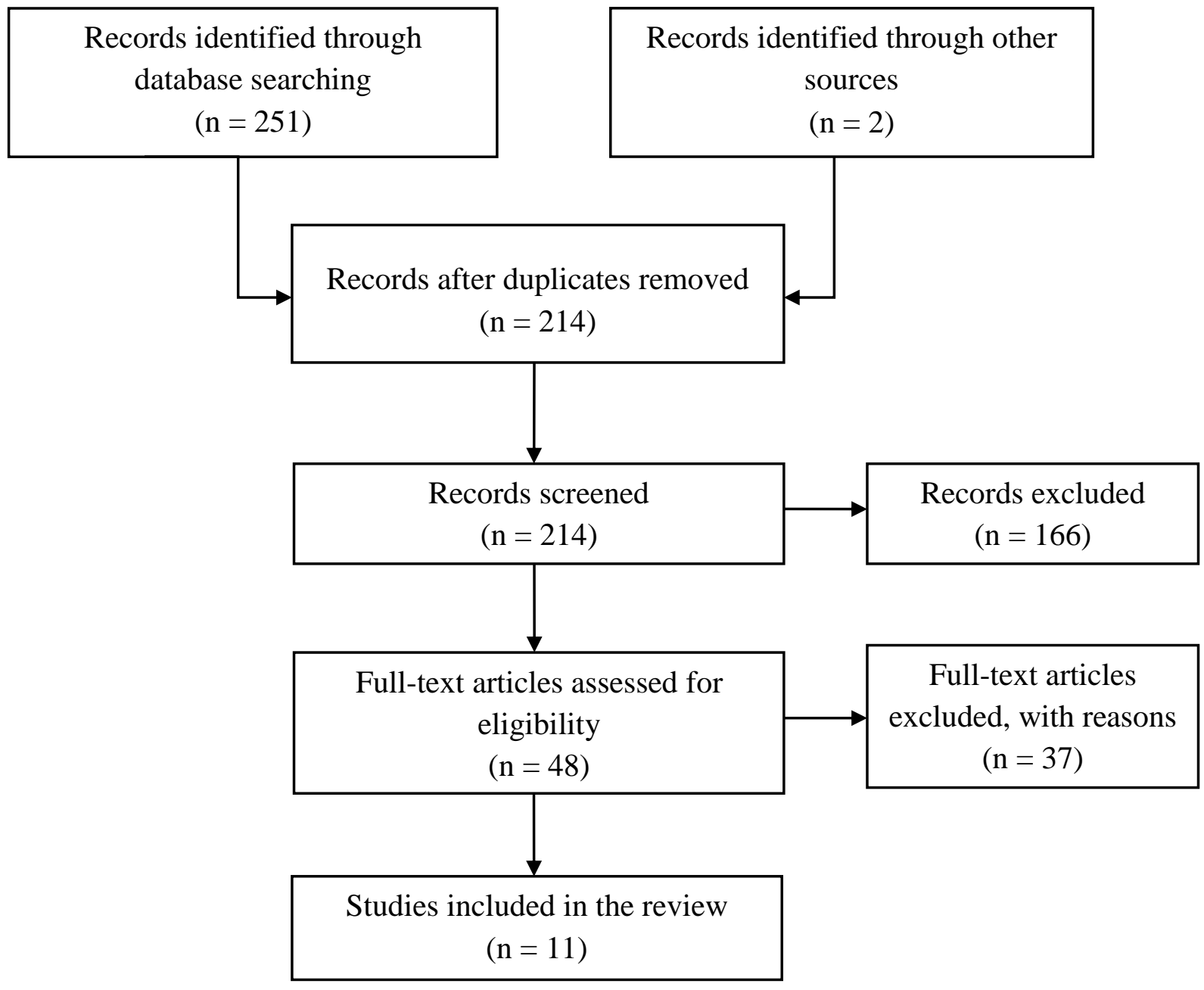

Figure1. Flowchart illustrating the different phases of the search and study selection

A total of 11 articles fulfilled the eligibility criteria. 
These papers are focused, particularly, on the roles of four design techniques in

208 learning tactical scenes in basketball $[2,13,65]$, soccer $[17,20,24,28,35,43,44]$, and 209 Australian football [66] through dynamic visualizations. One study [20] examined the effect of employing sequential presentation. Six studies [2, 13, 24, 35, 43, 44] tested the effect of using static visualizations. Four studies explored the effect of decreasing presentation speed $[17,35,65,66]$, and one study [28] examined the effect of using segmentation. These investigations were conducted within physical education or sports coaching domains. Most of these studies were designed to evaluate the effect of these design techniques on cognitive load, comprehension/recall accuracy (through a paper/pencil task), and game performance (during realistic situation) in order to obtain an indication of learning efficiency. The participants of three studies [2, 13, 65] were novices students (males and females) recruited from secondary school classes. They were aged between 15 and 16 years old. The participants of six studies [20, 24, 28, 35, 43, 44] were novices students (males) recruited from undergraduate university classes (aged between 22 and 29 years old), and experts players (aged between 24 and 29 years old) engaged with varied professional and semi-professional soccer football clubs located in French. The participants of one study [17] were sub-experts players (aged between 13 and 14 years old) engaged with teams from the second division of the Tunisian football league. The participants of one study [66] were novices $\left(\mathrm{M}_{\mathrm{age}}=22.68\right.$ years, $\mathrm{SD}=4.05)$, sub-experts $\left(\mathrm{M}_{\mathrm{age}}=20.34\right.$ years, $\left.\mathrm{SD}=3.44\right)$ and experts $\left(\mathrm{M}_{\mathrm{age}}=22.19\right.$ years, $\mathrm{SD}=3.10$ ) Australian footballers (males).

Table 1 lists the type of design technique, authors and year of publication, domain, type of dynamic visualizations, type of depicted knowledge, study sample, dependent variables, and study outcomes. 
230 Table1. Roles of design techniques in learning tactical scenes of play through dynamic visualizations: overview of the analyzed papers

\begin{tabular}{|c|c|c|c|c|c|c|c|}
\hline $\begin{array}{c}\text { Design } \\
\text { techniques }\end{array}$ & Source & Domain & $\begin{array}{c}\text { Dynamic } \\
\text { visualization }\end{array}$ & $\begin{array}{c}\text { Depicted } \\
\text { knowledge }\end{array}$ & Sample & $\begin{array}{c}\text { Dependant } \\
\text { variables }\end{array}$ & Study outcomes \\
\hline \multirow[t]{4}{*}{$\begin{array}{l}\text { Sequential } \\
\text { presentation }\end{array}$} & Khacharem et al. [20] & Soccer & Animation & Descriptive & $\begin{array}{l}\text { Novices } \\
\text { Experts }\end{array}$ & Recall accuracy & $\begin{array}{l}\text { For Novices } \\
\text { Sequential }>\text { concurrent } \\
\text { For experts } \\
\text { Sequential = concurrent }\end{array}$ \\
\hline & & & & & & Mental Effort & $\begin{array}{l}\text { For Novices } \\
\text { Sequential }<\text { concurrent } \\
\text { For experts } \\
\text { Sequential }>\text { concurrent }\end{array}$ \\
\hline & & & & & & $\begin{array}{l}\text { Number of } \\
\text { repetition }\end{array}$ & $\begin{array}{l}\text { For Novices } \\
\text { Sequential = concurrent } \\
\text { For experts } \\
\text { Sequential = concurrent }\end{array}$ \\
\hline & & & & & & Learning Efficiency & $\begin{array}{l}\text { For Novices } \\
\text { Sequential }>\text { concurrent } \\
\text { For experts } \\
\text { Sequential }<\text { concurrent }\end{array}$ \\
\hline \multirow[t]{2}{*}{$\begin{array}{c}\text { Static } \\
\text { visualizations }\end{array}$} & Khacharem et al. [43] & Soccer & Animation & Descriptive & $\begin{array}{l}\text { Novices } \\
\text { Experts }\end{array}$ & Mental Effort & $\begin{array}{l}\text { For Novices } \\
\text { Series of pictures }> \\
\text { Animation > Combined } \\
\text { For Experts } \\
\text { Animation < Series of } \\
\text { pictures < Combined }\end{array}$ \\
\hline & & & & & & Recall-Performance & $\begin{array}{l}\frac{\text { For Novices }}{\text { Animation }=\text { Series of }} \\
\text { pictures }<\text { Combined }\end{array}$ \\
\hline
\end{tabular}

.

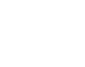


Khacharem et al. [44]
Soccer

Animation
Novices

Experts

\section{For Experts}

Animation $>$ Series of pictures $>$ Combined

Number of

repetitions

\section{For Novices}

Series of pictures $>$

Animation $>$ Combined

For Experts

Animation $<$ Series of

pictures $<$ Combined

Learning Efficiency For Novices

Series of pictures $>$

Animation $>$ Combined

For Experts

Animation $>$ Series of

pictures $>$ Combined

Recall accuracy

\section{For Novices}

Animation < Series of

pictures without tracing <

Series of pictures with tracing

For experts

Animation $=$ Series of

pictures without tracing $=$

Series of pictures with tracing

Mental Effort

\section{For Novices}

Series of pictures with tracing

$<$ Animation $=$ Series of pictures without tracing

\section{For experts}

Animation $<$ Series of

pictures without tracing $=$

Series of pictures with tracing 
Khacharem et al. [35]

\section{Soccer}

An Animatio

\section{Descriptive Novices Experts}

\section{Number of}

Repetitions

\section{For Novices}

Series of pictures with tracing

$<$ Animation $=$ Series of pictures without tracing

\section{For experts}

Animation $=$ Series of

pictures without tracing =

Series of pictures with tracing

Learning Efficiency For Novices

Animation $<$ Series of pictures without tracing <

Series of pictures with tracing For experts

Animation $>$ Series of

pictures without tracing $=$

Series of pictures with tracing

Recall accuracy

For Novices

Animation $=$ Picture

For Experts

Animation > Picture

Time on immediate recall test

For Novices

Animation $>$ Picture

For Experts

Animation $=$ Picture

Mental Effort

\section{For Novices}

Animation $>$ Picture

For Experts

Animation < Picture

Number of

For Novices

repetitions 
Khacharem et al. [24] Soccer

\section{Performance}

Time on delayed recall test

\section{Mental Effort}

Delayed recall accuracy

\section{Learning Efficiency}

For Experts

Animation < Picture

\section{For Novices}

Animation < Picture

For Experts

Animation > Picture

For Novices

Animation < Picture

For Experts

Animation > Picture

For Novices

Animation > Picture

For Experts

Animation $=$ Picture

For low content complexity

Animation $=$ diagram

For high content complexity

Animation < diagram

For low content complexity

Animation < diagram

For high content complexity

Animation $=$ diagram

Learning Efficiency For low content complexity Animation > diagram

For high content complexity

Animation < diagram 


\begin{tabular}{|c|c|c|c|c|c|c|c|}
\hline & & & & & & Comprehension & Video $>$ Series of pictures \\
\hline & & & & & & Game performance & Video $>$ Series of pictures \\
\hline & Rekik et al. [13] & Basketball & Video & $\begin{array}{l}\text { Motor } \\
\text { skills }\end{array}$ & Novices & Cognitive load & $\begin{array}{l}\text { For low content complexity } \\
\text { Video }=\text { Series of pictures } \\
\text { For medium } / \text { high contents } \\
\frac{\text { complexity }}{\text { Video }<\text { Series of pictures }}\end{array}$ \\
\hline & & & & & & Comprehension & $\begin{array}{l}\text { For low content complexity } \\
\text { Video }=\text { Series of pictures } \\
\text { For medium/high contents } \\
\text { complexity } \\
\text { Video }>\text { Series of pictures }\end{array}$ \\
\hline & & & & & & Game performance & $\begin{array}{l}\text { For low content complexity } \\
\text { Video }=\text { Series of pictures } \\
\text { For medium/high contents } \\
\text { complexity } \\
\text { Video }>\text { Series of pictures }\end{array}$ \\
\hline Segmentation & Khacharem et al. [28] & Soccer & Animation & Descriptive & $\begin{array}{l}\text { Novices } \\
\text { Experts }\end{array}$ & Recall accuracy & $\begin{array}{l}\frac{\text { For Novices }}{\text { Continuous }}=\text { Macro-step }= \\
\text { Micro-step } \\
\text { For experts } \\
\text { Continuous }<\text { Macro-step }< \\
\text { Micro-step }\end{array}$ \\
\hline & & & & & & Mental Effort & $\begin{array}{l}\text { For Novices } \\
\text { Continuous }>\text { Macro-step }> \\
\text { Micro-step } \\
\text { For experts } \\
\text { Continuous }>\text { Macro-step = } \\
\text { Micro-step }\end{array}$ \\
\hline
\end{tabular}




\begin{tabular}{|c|c|c|c|c|c|c|c|}
\hline \multirow[t]{3}{*}{$\begin{array}{l}\text { Decreasing } \\
\text { presentation } \\
\text { speed }\end{array}$} & Lorains et al. [66] & $\begin{array}{l}\text { Australian } \\
\text { football }\end{array}$ & Video & $\begin{array}{c}\text { Motor } \\
\text { skills }\end{array}$ & $\begin{array}{l}\text { Novices } \\
\text { Sub-Experts } \\
\text { Experts }\end{array}$ & Decision accuracy & $\begin{array}{l}\frac{\text { For Novices and Sub-Experts }}{\text { low speed = Normal speed }<} \\
\text { high speeds } \\
\text { For Experts } \\
\text { high speeds }>\text { Normal speed } \\
=\text { low speed }\end{array}$ \\
\hline & Khacharem et al. [35] & Soccer & Animation & Descriptive & $\begin{array}{l}\text { Novices } \\
\text { Experts }\end{array}$ & Recall accuracy & $\begin{array}{l}\frac{\text { For Novices }}{\text { High speed }}=\text { Normal speed }< \\
\text { low speed } \\
\text { For Experts } \\
\text { High speed }=\text { Normal speed }= \\
\text { low speed }\end{array}$ \\
\hline & & & & & & $\begin{array}{l}\text { Time on immediate } \\
\text { recall test }\end{array}$ & $\begin{array}{l}\frac{\text { For Novices }}{\text { High speed }>\text { Normal speed }>} \\
\text { low speed } \\
\text { For Experts } \\
\text { High speed }<\text { low speed }= \\
\text { Normal speed }\end{array}$ \\
\hline
\end{tabular}

Number of

repetition

Learning Efficiency

\section{Continuous < Macro-step < \\ Micro-step}

For experts

Continuous $<$ Macro-step $=$

Micro-step

Sub-Experts

high speeds > Normal speed

High speed $=$ Normal speed $<$

low speed

High speed $=$ Normal speed $=$

Normal speed 
Mental Effort

Number of

repetitions

Learning Effici
Delayed recall
accuracy

accuracy

Time on delayed recall test
For Novices

High speed $>$ Normal speed $>$

low speed

For Experts

High speed $=$ Normal speed $<$ low speed

For Novices

High speed > Normal speed > low speed

For Experts

High speed $=$ Normal speed $=$ low speed

For Novices

High speed $<$ Normal speed < low speed

For Experts

High speed $=$ Normal speed $>$ low speed

\section{For Novices}

High speed $=$ Normal speed $<$ low speed

For Experts

High speed $=$ Normal speed $=$ low speed

\section{For Novices}

High speed $=$ Normal speed $<$

low speed

For Experts

High speed $<$ Normal speed $=$ low speed 


\begin{tabular}{|c|c|c|c|c|c|c|}
\hline \multirow[t]{3}{*}{ Jarraya et al. [65] } & Basketball & Video & $\begin{array}{l}\text { Motor } \\
\text { skills }\end{array}$ & Novices & Mental Effort & $\begin{array}{l}\text { For low content complexity } \\
\text { Normal speed = low speed } \\
\text { For medium/high contents } \\
\text { complexity } \\
\text { Normal speed < low speed }\end{array}$ \\
\hline & & & & & Game performance & $\begin{array}{l}\frac{\text { For low content complexity }}{\text { Normal speed }=\text { low speed }} \\
\text { For medium/high contents } \\
\frac{\text { complexity }}{\text { Normal speed }<\text { low speed }}\end{array}$ \\
\hline & & & & & Learning Efficiency & $\begin{array}{l}\text { For low content complexity } \\
\text { Normal speed }=\text { low speed } \\
\text { For high content complexity } \\
\text { Normal speed }<\text { low speed }\end{array}$ \\
\hline \multirow[t]{3}{*}{ Rekik et al. [17] } & Soccer & Animation & Descriptive & Sub-Experts & Mental Effort & $\begin{array}{l}\text { For low content complexity } \\
\text { Normal speed }=\text { low speed } \\
\text { For high content complexity } \\
\text { Normal speed }>\text { low speed }\end{array}$ \\
\hline & & & & & Comprehension & $\begin{array}{l}\text { For low content complexity } \\
\text { Normal speed = low speed } \\
\text { For high content complexity } \\
\text { Normal speed < low speed }\end{array}$ \\
\hline & & & & & Learning Efficiency & $\begin{array}{l}\text { For low content complexity } \\
\text { Normal speed }=\text { low speed } \\
\text { For high content complexity } \\
\text { Normal speed < low speed }\end{array}$ \\
\hline
\end{tabular}




\subsection{Main findings}

Firstly, the reviewed articles revealed that the effectiveness of the four identified design techniques depend upon the level of learners' expertise when learning soccer scenes through animations showing descriptive knowledge. Indeed, it was observed that using static visualizations, employing sequential presentation, using segmentation, and decreasing presentation speed are effective only for less knowledgeable learners (i.e., novices), but they become ineffective for more knowledgeable learners (i.e., experts). Secondly, the present literature review showed that the effectiveness of using static visualizations, as design technique, instead of dynamic visualizations showing tactical scenes depend upon the type of the depicted knowledge (i.e., motor knowledge or descriptive knowledge), particularly for novice learners. In fact, it has been observed that replacing animations portraying descriptive knowledge with a series of static pictures or diagrams induce positive effects when learning soccer scenes among less knowledgeable learners. Conversely, using a series of static pictures instead of realistic videos portraying motor skills induce negative effects when learning basketball scenes among novice secondary school students. Thirdly, the reviewed papers demonstrate that the effectiveness of certain design techniques (i.e., using static visualizations, and decreasing presentation speed) depend upon the level of content complexity, especially for novice learners. In this context, it has been established that replacing a soccer animation with an arrows-based diagram induce positive effects on learning complex soccer scene of play (i.e., with high content complexity), but negative effects on learning simple soccer scene of play (i.e., with low content complexity). Moreover, using a series of static pictures instead of realistic videos portraying motor skills in basketball induce similar effects on learning when the content complexity was low, and negative effects on learning when the content complexity was medium and/or high. Furthermore, it was found that the instructional benefits of decreasing presentation speed of animations showing 
257 descriptive knowledge in soccer or realistic videos showing motor skills in basketball were 258 present only when studying medium or high levels of content complexity.

259 Table 2 provides a summary of the suggested design techniques in order to improve 260 learning tactical scenes of play through dynamic visualizations, as a function of these 261 moderator factors. 
262 Table2. Suggested design techniques to improve learning of tactical scenes of play through dynamic visualizations

\begin{tabular}{ccccc}
\hline $\begin{array}{c}\text { Type of dynamic } \\
\text { visualization }\end{array}$ & $\begin{array}{c}\text { Type of depicted } \\
\text { knowledge }\end{array}$ & $\begin{array}{c}\text { Level of content } \\
\text { complexity }\end{array}$ & Suggested design technique & Addressed to \\
\hline & & Sequential presentation & Novices \\
& Descriptive & High & Static visualizations & Novices \\
Animation & & Secreasing presentation speed & Novices / sub-Experts \\
& & Segmentation (Macro-step) & Novices / Experts \\
Video & Motor skills & Medium / High & Decreasing presentation speed & Novices \\
\hline
\end{tabular}




\section{Discussion}

This paper reviews a series of experimental studies examining the roles of different design techniques (without adding any oral/written explanations) in learning tactical scenes of play through dynamic visualizations. The literature search strategies yielded a final pool of eleven possible papers. These articles are interested to the role of four design techniques (using static visualizations, employing sequential presentation, using segmentation, and decreasing presentation speed) on tactical learning in basketball, soccer, and Australian football. Overall, research into the instructional and/or cognitive effects of these design techniques has obtained discrepant results. In fact, the roles of these design techniques in learning tactical scenes from dynamic visualizations depends/varies as a function of the level of learners' expertise, type of depicted knowledge, and level of content complexity.

\subsection{Level of learners' expertise}

The current state of the literature indicated that learner prior knowledge is a significant factor that could moderate the effectiveness of all identified design techniques (i.e., using static visualizations, employing segmentation, using sequential presentation, and decreasing presentation speed) when learning tactical scenes of play through dynamic visualizations, especially via animations showing descriptive knowledge.

In this framework, Khacharem et al. [20] found that the effect of using sequential presentation was moderated by the level of players' expertise when learning soccer drill from an animation. In this study, participants were invited to complete a recall-reconstruction test and to rate their invested mental effort after studying a concurrent or sequential presentation of a soccer animation. For novice players, the sequential presentation produced better learning outcomes. Conversely, expert players performed better after studying the concurrent presentation. 
Moreover, the effective use of the segmentation technique was also moderated by the level of learners' expertise when studying complex soccer scenes from animations. Khacharem et al. [28] tested the effect of two types of segmentation (macro-step and microstep) on learning soccer attacking drills. Even though results demonstrated positive effect of the macro-step segmentation among all players, novices benefited more from micro-step segmentation than from macro-step segmentation, while experts performed at the same level with both forms of segmentation.

Furthermore, Khacharem et al. [43, 44] investigated the effects of expertise on perceived cognitive load and performance resulting from studying soccer scene either through an animation or via a series of static pictures. The results showed that novice players achieved higher performance outcomes after studying static pictures. However, expert players performed better after studying instructional animations. Similarly, Khacharem et al. [35] found an interaction between levels of learner expertise and the usefulness of replacing an animation with a static picture in studying a soccer playing system. According to this study, displaying a static picture to novice players is more helpful for learning than displaying an animation. Conversely, learning from a continuous animation is more beneficial for expert players: they attained the higher level of performance with the same time on the immediate recall-test, needed lower number of repetitions, and invested less mental effort.

Additionally, it was established that learners' prior knowledge should be taken into consideration when decreasing animation speed. For example, Khacharem et al. [35] examined the effect of three presentation speed (high vs. normal vs. low) on learning soccer scene among novices and expert players. The study reported mixed effects for the use of these animations, when considering the level of learners' expertise. Indeed, novice players achieved higher recall scores, needed a lower number of repetitions and invested less mental effort when the animations were played at a low speed than when they were played at a normal or 
high speed. However, expert players had to invest less mental effort to attain the same level of performance with the same number of repetitions, when the animations were displayed at a high or normal speed than when they were displayed at a low speed.

According to these studies, the interaction between the effectiveness of design techniques and the levels of learners' expertise when learning from dynamic visualizations is mainly due to "the expertise reversal effect" [for a review, see 45-48]. Accordingly, learning from animations depends not only on how the information is presented, but also on the quantity of the learner prior knowledge in the domain. It is well known that prior knowledge is stored in long term memory as cognitive schemas, through experience and deliberate practice $[44,49]$. The development of domain-specific knowledge can effectively reduce WM overload by assembling a large amount of information elements into a single unit. As a result, experienced learners were able to deal with complex dynamic visualizations, by identifying the crucial aspects and ignore the unimportant ones [50-52]. Consequently, design techniques that are optimal and effective for less knowledgeable learners may become ineffective and hinder learning for more knowledgeable learners, and vice versa [35, 45, 46, 47].

\subsection{Type of depicted knowledge}

It has been established that the type of knowledge depicted in dynamic visualizations (i.e., motor knowledge or descriptive knowledge) could moderate the effectiveness of one of the above-mentioned design techniques (i.e., using static visualizations) when learning tactical scenes, particularly for novice learners.

On one hand, Khacharem and colleagues $[43,44]$ found that replacing animations with a series of static pictures is an effective strategy for learning soccer attacking drills, especially for novice soccer players. Similarly, it was established that using a static picture representing three key stages of a soccer animation is more beneficial for learning: novice players attained the same level of performance with less time on the immediate recall-test, with lower number 
of repetitions, and with lower investment of mental effort [35]. In the same vein, Khacharem et al. [24] investigate the instructional effectiveness of using a soccer animation in comparison to using a static diagram. The results demonstrated that novice players benefited more from studying a static presentation than from studying an animated presentation: they achieved the same level of comprehension with lower investment of mental effort. As mentioned in the introduction, using static instead of dynamic visualizations may decrease the extraneous cognitive load investment by allowing learners to benefit from sufficient time to identify and process relevant information and effectively integrate it in long term memory $[25,26]$. Moreover, using static visualizations, compared to dynamic representations, offer the possibility to revise and compare different parts of the display as frequently as desired [27].

One the other hand, evidence of positive effects of using static visualizations were not proved in comparison with using dynamic visualizations among novice learners, when it was about learning motor knowledge/skills. In this context, Rekik et al. [2] explored the effectiveness of video versus a series of static photographs on learning basketball tactical actions within physical education domain. Immediately after the learning phase, students were asked to indicate their cognitive load investment. Next, they were invited to perform a game understanding task and a game performance task. For all indicators, the results showed that learning from the video was more effective than learning from a series of photographs. These results are consistent with previous researches carried out in non-sporting domains, demonstrating the cognitive and instructional value of dynamic visualizations (as opposed to statics) involving various motor skills that require hand manipulations such as performing an emergency procedure [9], making origami shapes [53], constructing 3D Lego figures [54], and tying knots [5, 6, 55, and 56].

Following the neuroscience literature, the superiority of dynamic visualizations over statics when learning motor knowledge/skills is mainly due to the activation of the Mirror- 
363 Neuron System [57-60]. This system is originally identified in primates. It is a neuro-

364 physiological circuit distributed across the pre-motor cortex that is automatically activated 365 when someone is observing another person performing an action [58, 60]. Moreover, as 366 humans' actions are part of primary knowledge such as face recognition, learning from others, 367 and language, their acquisition is very easy and requires little cognitive effort [61]. Hence, 368 watching dynamic visualizations involving motor skills does not require excessive cognitive 369 resources, because humans are biologically evolved to effectively acquire such kind of 370 knowledge. The phenomenon of learning motor skills from dynamic visualizations compared 371 to statics was called "the human movement effect" [61].

\subsection{Level of content complexity}

Analysis of the selected articles showed that the level of content complexity (i.e., the number of interactive information elements) is a significant factor that could modulate the effectiveness of some design techniques (i.e., using static visualizations, decreasing presentation speed) when learning tactical game systems through dynamic visualizations, particularly for novice learners. The term "complexity" used in these experimental studies referred to the internal complexity of the playing systems that was associated with the intrinsic cognitive load [62]. In fact, the more complex scene of play is the situation that involves more players and more interactions between them [63, 64].

It was established that replacing an animation with an arrows-based diagram was efficacious only when studying complex soccer scene of play (i.e., with high content complexity). Indeed, novice players achieved the same level of comprehension with lower investment of mental effort. By contrast, participants learned more efficiently from the animation than from the static diagram when it is about a simple soccer scene [see 24]. Moreover, Rekik et al. [13] found that using a series of static pictures or a video had similar effects among novice participants when learning basketball scenes with low content 
complexity. However, for medium and high content complexity, the dynamic format had a clear advantage over the static format in terms of cognitive load investment and learning outcomes.

In addition, it was found that the instructional benefits of decreasing presentation speed of animations showing descriptive knowledge or videos showing motor skills were also affected by the level of content complexity. Rekik et al. [17] examined the effect of content complexity on learning from soccer animations presented either at normal or low speeds (i.e., 0.5 and 1.0 times normal speed). Their results revealed that while the decrease of presentation speed had no advantages when learning low-complexity content, sub-expert players profited more from the low than the normal presentation speed when learning high complexity content (based on the combination of comprehension and cognitive load scores). The same pattern of results was obtained when learning basketball tactical actions through videos [see 65]. Authors found that both speeds of presentation have similar effects when learning low content complexity. Conversely, for medium and high complexity contents, novice participants exposed to the slow-presentation speed learned more efficiently than those exposed to the normal-presentation speed.

These researchers referred usually to the cognitive load theory [14, 15] in order to explain the interaction between the effectiveness of design techniques and the levels of content complexity when learning from dynamic visualizations. Indeed, dynamic formats displaying contents with low levels of complexity led to easier learning, because learners had to consume less perceptual-cognitive resources to deal with both the transient nature of information and few numbers of interactive information elements. As a result, learners were not forced to integrate and maintain excessive information elements in working memory. Consequently, novice learners could benefit from videos or animations showing tactical scenes of play without running the risk of a potential cognitive overload. By contrast, dealing 
413 with more complex dynamic visualizations made learning difficult and consumed a large amount of perceptual-cognitive resources, as learners were asked to deal with the transient nature of information and to spatially split their attention among the excessive number of interactive elements [14]. Therefore, the use of the above-mentioned design techniques (except the use of static visualizations when learning motor skills; due to the human movement effect) might reduce these cognitive processing demands and improve novices' performance when learning tactical scenes of play through dynamic visualizations.

\section{Strengths and weaknesses}

As a first initiative, the present study offers a comprehensive coverage of the available literature and the careful appraisal of its quality, via the utilization of a wide range of key words (related to the relationships between dynamic visualizations, design techniques, and learning tactical scenes of play) searched through two globe databases. The current review demonstrated important practical implications for both coaches and physical education teachers using either animations or realistic video clips to communicate/explain tactical scenes. Indeed, the present review shows that adapting design techniques to the level of learners' expertise, type of depicted knowledge, and level of content complexity is a crucial part of effective learning. However, certain limitations should be kept in mind. First, the current review paper focused solely on experimental studies based on purely visual learning environment (i.e., without adding any oral/written explanations). Although this requirement was applied in order to avoid the occurrence of modality effect [41, 42], it would be worthwhile in future review to include studies based on multimedia learning environment. Second, the present literature review was interested specifically on tactical learning in team sports. More review papers are required to explore the roles of design techniques in learning from dynamic visualizations portraying actions/events in individual sports, such as gymnastic or weightlifting. 
438

439

440

441

442 Funding: We acknowledge financial support by Taipei Veterans General Hospital (\# V98E2-

443 014).

444 Conflicts of Interest: The authors declare no conflict of interest.

Author Contributions: G.R. conceptualized and planned the study. G.R. and Y.B. undertook the study selection and quality assessment. G.R., Y.B. and M.J. contributed in data interpretation. G.R. drafted the manuscript, which was critically reviewed by M.A.B., Y.S.C. and C.D.K. All authors have read and agreed to the published version of the manuscript.
445

446

447

448

449

450

451

452

453

454

455

456

457

458

459

460

461

462

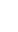

(

. 
463

464

465

466

467

468

469

470

471

472

473

474

475

476

477

478

479

480

481

482

483

484

485

486

\section{References}

1. Schnotz, W.; Lowe, R.K. A unified view of learning from animated and static graphics. In Learning with animation: Research implications for design, $1^{\text {st }}$ ed.; Lowe, R.K., Schnotz, W., Eds.; Cambridge: Cambridge University Press, New York, NY, 2008. pp. 304-356.

2. Rekik, G.; Khacharem, A.; Belkhir, Y.; Bali, N.; Jarraya, M. The instructional benefits of dynamic visualizations in the acquisition of basketball tactical actions. J. Comput. Assist. Learn. 2019, 35, 74-81.

3. Kriz, S.; Hegarty, M. Top-down and bottom-up influences on learning from animations. Int. J. Hum. Comput. Stud. 2007, 65, 911-930.

4. Kühl, T.; Scheiter, K.; Gerjets, P.; Edelmann, J. The influence of text modality on learning with static and dynamic visualizations. Comput. Hum. Behav. 2011, 27, 29-35.

5. Garland, T.B.; Sanchez, C.A. Rotational perspective and learning procedural tasks from dynamic media. Comput. Educ. 2013, 69, 31-37.

6. Boucheix, J.M.; Forestier, C. Reducing the transience effect of animations does not (always) lead to better performance in children learning a complex hand procedure. Comput. Hum. Behav. 2017, 69, 358-370.

7. Lowe, R.K. Interrogation of a dynamic visualization during learning. Learn. Instr. 2004, $14,257-274$.

8. Schnotz, W.; Bannert, M. Construction and interference in learning from multiple representation. Learn. Instr. 2003, 13, 141-156.

9. Arguel, A.; Jamet, E. (2009). Using video and static pictures to improve learning of procedural contents. Comput. Hum. Behav. 2009, 25 354-359.

10. Hegarty, M.; Kriz, S.; Cate, C. The roles of mental animations and external animations in understanding mechanical systems. Cogn. Instr. 2003, 21, 209-249. 
487

488

489

490

491

492

493

494

495

496

497

498

499

500

501

502

503

504

505

506

507

508

509

11. Pedra, A.; Mayer, R.E.; Albertin, A.L. Role of interactivity in learning from engineering animations. Appl. Cogn. Psychol. 2015, 29, 614-620.

12. Türkay, S. The effects of whiteboard animations on retention and subjective experiences when learning advanced physics topics. Comput. Educ. 2016, 98, 102-114.

13. Rekik, G.; Khacharem, A.; Belkhir, Y.; Bali, N.; Jarraya, M. The effect of visualization format and content complexity on acquisition of tactical actions in basketball. Learn. Motiv. 2019, 65, 10-19.

14. Sweller, J.; Van Merrienboer, J.J.; Paas, F.G. Cognitive architecture and instructional design. Educ. Psychol. Rev. 1998, 10, 251-296.

15. Sweller, J.; Ayres, P.; Kalyuga, S. Measuring cognitive load. In Cognitive load theory; Springer Nature: New York, NY, 2011; Volume 1, pp. 71-85.

16. Baddeley, A. Working memory: looking back and looking forward. Nat. Rev. Neurosci. 2003, 4, 829-839.

17. Rekik, G.; Belkhir, Y.; Mnif, M.; Masmoudi, L.; Jarraya, M. Decreasing the Presentation Speed of Animated Soccer Scenes Does Not Always Lead to Better Learning Outcomes in Young Players. Int. J. Hum-Comput. Int. 2020, 36, 717-724.

18. Leahy, W.; Sweller, J. Cognitive load theory, modality of presentation and the transient information effect. Appl. Cogn. Psychol. 2011, 25, 943-951.

19. Moreno, R.; Mayer, R. Interactive multimodal learning environments. Educ. Psychol. Rev. 2007, 19, 309-326.

20. Khacharem, A.; Zoudji, B.; Kalyuga, S.; Ripoll, H. The expertise reversal effect for sequential presentation in dynamic soccer visualizations. J. Sport. Exerc. Psychol. 2013, 35, 260-269. 
510

511

512

513

514

515

516

517

518

519

520

521

522

523

524

525

526

527

528

529

530

531

532

533

534

21. Paas, F.; Van Gerven, P.W.; Wouters, P. Instructional efficiency of animation: Effects of interactivity through mental reconstruction of static key frames. Appl. Cogn. Psychol. 2007, 21, 783-793.

22. Boucheix, J.M.; Schneider, E. Static and animated presentations in learning dynamic mechanical systems. Learn. Instr. 2009, 19, 112-127.

23. Castro-Alonso, J.C.; Ayres, P.; Wong, M.; Paas, F. Learning symbols from permanent and transient visual presentations: Don't overplay the hand. Comput. Educ. 2018, 116, 1-13.

24. Khacharem, A.; Zoudji, B.; Kalyuga, S. Perceiving versus inferring movements to understand dynamic events: The influence of content complexity. Psychol. Sport. Exerc. 2015, 19, 70-75.

25. Mayer, R.E.; DeLeeuw, K.E.; Ayres, P. Creating retroactive and proactive interference in multimedia learning. Appl. Cogn. Psychol. 2007, 21, 795-809.

26. Scheiter, K.; Gerjets, P.; Catrambone, R. Making the abstract concrete: Visualizing mathematical solution procedures. Comput. Hum. Behav. 2006, 22, 9-25.

27. Hegarty, M. Dynamic visualizations and learning: Getting to the difficult questions. Learn. Instr. 2004, 14, 343-351.

28. Khacharem, A.; Spanjers, I.A.; Zoudji, B.; Kalyuga, S.; Ripoll, H. Using segmentation to support the learning from animated soccer scenes: An effect of prior knowledge. Psychol. Sport. Exerc. 2013, 14, 154-160.

29. Spanjers, I.A.; Wouters, P.; Van Gog, T.; Van Merrienboer, J.J. An expertise reversal effect of segmentation in learning from animated worked-out examples. Comput. Hum. Behav. 2011, 27, 46-52.

30. Spanjers, I.A.; van Gog, T.; Wouters, P.; van Merriënboer, J.J. Explaining the segmentation effect in learning from animations: The role of pausing and temporal cueing. Comput. Educ. 2012, 59, 274-280. 
535

536

537

538

539

540

541

542

543

544

545

546

547

548

549

550

551

552

553

554

555

556

557

31. Boucheix, J.M.; Lowe, R.K.; Putri, D.K.; Groff, J. Cueing animations: Dynamic signaling aids information extraction and comprehension. Learn. Instr. 2013, 25, 71-84.

32. De Koning, B.B.; Tabbers, H.K.; Rikers, R.M.; Paas, F. Towards a framework for attention cueing in instructional animations: Guidelines for research and design. Educ. Psychol. Rev. 2009, 21, 113-140.

33. Ayres, P.; Paas, F. Making instructional animations more effective: A cognitive load approach. Appl. Cogn. Psychol. 2007, 21, 695-700.

34. Castro-Alonso, J.C.; Ayres, P.; Paas, F. Dynamic visualizations and motor skills. In Handbook of human centric visualization; Huang, W., Eds.; Springer, New York, NY, 2014. pp. 551-580.

35. Khacharem, A.; Zoudji, B.; Spanjers, I.A.; Kalyuga, S. Improving learning from animated soccer scenes: Evidence for the expertise reversal effect. Comput. Hum. Behav. 2014, 35, 339-349.

36. Lowe, R. Changing perceptions of animated diagrams. In International Conference on Theory and Application of Diagrams, 4th International Conference, Diagrams 2006, Stanford, CA, USA, 2006; Springer: Berlin, Heidelberg, 2006, pp. 168-172.

37. Meyer, K.; Rasch, T.; Schnotz, W. Effects of animation's speed of presentation on perceptual processing and learning. Learn. Instr. 2010, 20, 136-145.

38. Chen, C.Y.; Yen, P.R. Learner control, segmenting, and modality effects in animated demonstrations used as the before-class instructions in the flipped classroom. Interact. Learn. Environ. 2019, 1-15.

39. Schwan, S.; Riempp, R. The cognitive benefits of interactive videos: learning to tie nautical knots. Learn. Instr. 2004, 14, 293-305. 
558

559

560

561

562

563

564

565

566

567

568

569

570

571

572

573

574

575

576

577

578

579

580

581

40. Moher, D.; Liberati, A.; Tetzlaff, J.; Altman, D.G. Preferred reporting items for systematic reviews and meta-analyses: The PRISMA statement. J. Clin. Epidemiol.2009, 62, 1006101

41. Mayer, R.E. Multimedia learning, 2nd ed.; Cambridge: Cambridge University Press, New York, NY, 2001, pp. 66.

42. Mayer, R. E. Research-based principles for learning with animation. In Learning with animation: Research implications for design, 1st ed.; Lowe, R.K., Schnotz, W., Eds.; Cambridge: Cambridge University Press, New York, NY, 2008. pp. 30-48.

43. Khacharem, A.; Zoudji, B.; Ripoll, H. Effect of presentation format and expertise on attacking-drill memorization in soccer. J. Appl. Sport. Psychol. 2013, 25, 234-248.

44. Khacharem, A.; Zoudji, B.; Kalyuga, S.; Ripoll, H. Developing tactical skills through the use of static and dynamic soccer visualizations: An expert-nonexpert differences investigation. J. Appl. Sport Psychol. 2013, 25, 326-340.

45. Kalyuga, S. Prior knowledge principle in multimedia learning. In The Cambridge handbook of multimedia learning, $1^{\text {st }}$ ed.; Mayer, R.E., Eds.; Cambridge: Cambridge University Press, New York, NY, 2005. pp. 325-337.

46. Kalyuga, S. Assessment of learners' organised knowledge structures in adaptive learning environments. Appl. Cogn. Psychol. 2006, 20, 333-342.

47. Kalyuga, S. Expertise reversal effect and its implications for learner-tailored instruction. Educ. Psychol. Rev. 2007, 19, 509-539.

48. Kalyuga, S.; Ayres, P.; Chandler, P.; Sweller, J. The Expertise Reversal Effect. Educ. Psychol. 2003, 38, 23-31.

49. Ericsson, K.A.; Lehmann, A.C. Expert and exceptional performance: Evidence of maximal adaptation to task constraints. Annu. Rev. Psychol. 1996, 47, 273-305. 
582

583

584

585

586

587

588

589

590

591

592

593

594

595

596

597

598

599

600

601

602

603

604

605

50. Ayres, P.; Kalyuga, S.; Marcus, N.; Sweller, J. The conditions under which instructional animation may be effective. In an International Workshop and Mini-conference, Open University of the Netherlands, Heerlen, The Netherlands, 2005.

51. Kalyuga, S. Relative effectiveness of animated and static diagrams: An effect of learner prior knowledge. Comput. Hum. Behav. 2008, 24, 852-861.

52. Gegenfurtner, A.; Lehtinen, E.; Säljö, R. Expertise differences in the comprehension of visualizations: A meta-analysis of eye-tracking research in professional domains. Educ. Psychol. Rev. 2011, 23, 523-552.

53. Wong, A.; Leahy, W.; Marcus, N.; Sweller, J. Cognitive load theory, the transient information effect and e-learning. Learn. Instr. 2012, 22, 449-457.

54. Castro-Alonso, J.C.; Ayres, P.; Paas, F. Animations showing Lego manipulative tasks: Three potential moderators of effectiveness. Comput. Educ. 2015, 85, 1-13.

55. Ayres, P.; Marcus, N.; Chan, C.; Qian, N. Learning hand manipulative tasks: When instructional animations are superior to equivalent static representations. Comput. Hum. Behav. 2009, 25, 348-353.

56. Marcus, N.; Cleary, B.; Wong, A.; Ayres, P. Should hand actions be observed when learning hand motor skills from instructional animations?. Comput. Hum. Behav. 2013, 29, 2172-2178.

57. Buccino, G.; Binkofski, F.; Riggio, L. The mirror neuron system and action recognition. Brain. Lang. 2004, 89, 370-376.

58. Rizzolatti, G.; Craighero, L. The mirror-neuron system. Annu. Rev. Neurosci. 2004, 27, 169-192.

59. Turella, L.; Pierno, A.C.; Tubaldi, F.; Castiello, U. Mirror neurons in humans: Consisting or confounding evidence?. Brain. Lang. 2009, 108, 10-21. 
606

607

608

609

610

611

612

613

614

615

616

617

618

619

620

621

622

623

624

625

60. Van Gog, T.; Paas, F.; Marcus, N.; Ayres, P.; Sweller, J. The mirror neuron system and observational learning: Implications for the effectiveness of dynamic visualizations. Educ. Psychol. Rev. 2009, 21(1), 21-30.

61. Paas, F.; Sweller, J. An evolutionary upgrade of cognitive load theory: Using the human motor system and collaboration to support the learning of complex cognitive tasks. Educ. Psychol. Rev. 2012, 24, 27-45.

62. Pollock, E.; Chandler, P.; Sweller, J. Assimilating complex information. Learn. Instr. 2002, 12, 61-86.

63. Raab, M. T-ECHO: Model of decision making to explain behavior in experiments and simulations under time pressure. Psychol. Sport. Exerc. 2002, 3, 151-171.

64. Raab, M. Decision making in sports: Influence of complexity on implicit and explicit learning. Int. J. Sport. Exerc. Psychol. 2003, 1, 406-433.

65. Jarraya, M.; Rekik, G.; Belkhir, Y.; Chtourou, H.; Nikolaidis, P.T.; Rosemann, T.; Knechtle, B. Which presentation speed is better for learning basketball tactical actions through video modeling examples? The influence of content complexity. Front. Psychol. 2019, 10 .

66. Lorains, M.; Ball, K.; MacMahon, C. Expertise differences in a video decision-making task: Speed influences on performance. Psychol. Sport. Exerc. 2013, 14, 293-297. 
